\title{
CHARACTER MODULES, DIMENSION AND PURITY
}

\author{
by DAVID J. FIELDHOUSE
}

(Received 19 October, 1970; revised 11 June, 1971)

1. Introduction. In this paper we use the Bourbaki conventions for rings and modules: all rings are associative but not necessarily commutative and have an identity element; all modules are unital.

For any $A$-module $M$, let $M^{*}=\operatorname{Hom}_{\mathrm{Z}}(M, \mathbf{Q} / \mathbf{Z})$ denote the character module of $M$, where $\mathbf{Q}$ denotes the rationals and $\mathbf{Z}$ the integers. Then ${ }^{*}$ is an exact contravariant zeroreflecting (i.e. $M=0$ if and only if $M^{*}=0$ ) functor from the category of left $A$-modules to the category of right $A$-modules. Details may be found in Lambek [6], whose results in [5] we extend.

We introduce the absolute pure dimension (apd), relate it to the weak and injective dimensions, denoted wd and injd respectively, in terms of character modules, and deduce some results on the corresponding (left) global dimensions, denoted lapgl, wgl, and lgl respectively. We conclude with some remarks on absolute purity. Basic facts on purity and regularity can be found in the author's paper [4]. Facts on dimensions used can be found in CartanEilenberg [2].

2. The dimension theorems. For any module $M$, we define apd $M$ to be the least integer $n$ such that $\operatorname{Ext}^{n+1}(N, M)=0$ for all finitely presented modules $N$. If no such integer exists, apd $M$ is defined to be infinite. Clearly apd $M \leqq \operatorname{injd} M$ for all $M$, and equality holds if $A$ is noetherian, since injd $M \leqq n$ if and only if $\operatorname{Ext}^{n+1}(A / I, M)=0$ for all ideals $I$ of $A$. Later we shall show the converse that, if apd $M=\operatorname{injd} M$ for all $M$, then $A$ must be noetherian.

Theorem 2.1. For all $M$, we have apd $M^{*}=\operatorname{wd} M=\operatorname{injd} M^{*}$.

Proof. Since $\mathbf{Q} / \mathbf{Z}$ is $\mathbf{Z}$-injective, we have $\operatorname{Ext}^{n}\left(N, M^{*}\right)=\left(\operatorname{Tor}^{n}(N, M)\right)^{*}$ for all $N, M$ and all $n \geqq 0$. (See Cartan-Eilẹnberg [2], p. 120.) Hence

$$
\begin{aligned}
\operatorname{apd} M^{*} \leqq n & \Leftrightarrow \operatorname{Ext}^{n+1}\left(N, M^{*}\right)=0 \quad \text { for all finitely presented } N \\
& \Leftrightarrow \operatorname{Tor}^{n+1}(N, M)=0 \text { for all finitely presented } N \\
& \Leftrightarrow \operatorname{wd} M \leqq n \\
& \Leftrightarrow \operatorname{Tor}^{n+1}(N, M)=0 \text { for all } N \\
& \Leftrightarrow \operatorname{Ext}^{n+1}\left(N, M^{*}\right)=0 \text { for all } N \\
& \Leftrightarrow \operatorname{injd} M^{*} \leqq n .
\end{aligned}
$$

COROLlaRY.

$$
\text { wgl } A \leqq \operatorname{lapgl} A \leqq \operatorname{lgl} A \text {. }
$$

We recall that a ring $A$ is left coherent if and only if every finitely presented left $A$-module 
is coherent, which means that all finitely generated submodules are finitely presented. For details see Bourbaki [1, pp. 62-63].

For the following result we shall use the fact that over a coherent ring every finitely presented module has a resolution by finitely generated free modules.

TheOREM 2.2. For left coherent rings, wd $M^{*}=$ apd $M$, for all left $A$-modules $M$.

Proof. Since $A$ is left coherent, we have, by Cartan-Eilenberg [2, pp. 120-121], $\operatorname{Tor}^{n}\left(M^{*}, N\right)=\left(\operatorname{Ext}^{n}(N, M)\right)^{*}$ for all $M$, all finitely presented $N$ and all $n \geqq 0$; and

$$
\text { apd } \begin{aligned}
M \leqq n & \Leftrightarrow \operatorname{Ext}^{n+1}(N, M)=0 \quad \text { for all finitely presented } N, \\
& \Leftrightarrow \operatorname{Tor}^{n+1}\left(M^{*}, N\right)=0 \text { for all finitely presented } N, \\
& \Leftrightarrow \mathrm{wd} M^{*} \leqq n .
\end{aligned}
$$

Hence wd $M^{*}=\operatorname{apd} M$.

Corollary 1. For left coherent rings, $\operatorname{lapgl} A=\operatorname{wgl} A$.

COROLlaRY 2. For coherent rings, apd $M$ is the least integer $n$ such that $\operatorname{Ext}^{n+1}(A / I, M)=0$ for all finitely generated ideals $I$ of $A$.

Proof. Use Theorems 2.2 and 2.3.

TheOrem 2.3. For any left $A$-module $M$ we have: $w d M \leqq n$ if and only if $\operatorname{Tor}^{n+1}(A / I, M)$ $=0$ for all finitely generated right ideals $I$ of $A$.

Proof. By definition wd $M \leqq n$ if and only if $\operatorname{Tor}^{n+1}(N, M)=0$ for all right modules $N$. Since Tor ${ }^{n}$ commutes with direct limits for all $n$ and since every module is the direct limit of its finitely generated submodules, we can immediately reduce the problem to the case where $N$ is finitely generated. By induction on the number of generators of $N$ we can reduce to the cyclic module case: $A / I, I$ a right ideal of $A$. Since $I$ is the direct limit of finitely generated right ideals, we get the desired result.

COROLLARY 1. wgl $A \leqq 1+\sup$ wd $I$ with the sup taken over all finitely generated left (or right) ideals of $A$. If $\mathrm{wgl} A \geqq 1$ we have equality. Hence $\mathrm{wgl} A \leqq 1$ if and only if all finitely generated left (or right) ideals are flat.

Proof. By the theorem, wgl $A=\sup w d A / I$ with the sup taken over all finitely generated left (or right) ideals. But wd $(A / I)=1+\mathrm{wd} I$ unless wd $A / I=0$. If $\mathrm{wgl} A \geqq 1$, then $A$ is not regular; hence some $I$ is not pure in $A$ and $A / I$ is not flat.

CoRollary 2. A ring $A$ is left semihereditary if and only if it is left coherent and $\mathrm{wgl} A \leqq 1$.

Proof. Any finitely generated module is projective if and only if it is finitely presented and flat. Apply this result to any finitely left ideal of $A$.

3. Purity. A short exact sequence of left $A$-modules $E: 0 \rightarrow E_{1} \rightarrow E_{2} \rightarrow E_{3} \rightarrow 0$ is pure exact in the sense of Cohn [3] if and only if, for all (or equivalently for all finitely presented) 
right $A$-modules $M$, we have

$$
M \otimes E: 0 \rightarrow M \otimes E_{1} \rightarrow M \otimes E_{2} \rightarrow M \otimes E_{3} \rightarrow 0
$$

exact. We have shown in [4] that this is equivalent to $\operatorname{Hom}\left(N, E_{2}\right) \rightarrow \operatorname{Hom}\left(N, E_{3}\right)$ being epic for all finitely presented $N$.

A module is called absolutely pure if and only if it is pure in every overmodule; actually it suffices to test in the injective hull.

THEOREM 3.1. A module is absolutely pure if and only if apd $M=0$.

Proof. Let $I$ denote the injective hull of $M$. For every module $N$ we have an exact sequence:

$$
\operatorname{Hom}(N, I) \stackrel{\alpha_{N}}{\rightarrow} \operatorname{Hom}(N, I / M) \rightarrow \operatorname{Ext}(N, M) \rightarrow \operatorname{Ext}(N, I)=0 .
$$

Hence $\alpha_{N}$ is epic if and only if $\operatorname{Ext}(N, M)=0$.

Therefore $M$ is pure in $I$ if and only if $\operatorname{Ext}(N, M)=0$ for all finitely presented $N$, i.e., if and only if apd $M=0$.

Corollary. $A$ is left noetherian if and only if apd $M=\operatorname{injd} M$ for all left $A$-modules $M$.

Proof. We have discussed the "only if" part. The "if" part now follows from Maddox [7]. Note that the dimension zero case suffices.

\section{REFERENCES}

1. N. Bourbaki, Algebre commutative (Paris, 1961).

2. H. Cartan and S. Eilenberg, Homological algebra (Princeton, 1956).

3. P. M. Cohn, On the free product of associative rings I, Math. Z. 71 (1959), 380-398.

4. D. Fieldhouse, Pure theories, Math. Ann. 184 (1969), 1-18.

5. J. Lambek, A module is flat if and only if its character module is injective, Canad. Math. Bull. 7 (1964), 279-289.

6. J. Lambek, Lectures on rings and modules (Blaisdell, 1966).

7. B. Maddox, Absolutely pure modules, Proc. Amer. Math. Soc. 18 (1967), 155-158.

UNIVERSITY OF GUELPH

Guelph, Ontario, Canada 